\title{
Fractal and spectral analysis of fracture surfaces of elastomeric materials
}

\author{
D. Ait Aouit \& A. Ouahabi \\ Ecole polytechnique de Tours, Université Francois Rabelais, France
}

\begin{abstract}
Crack path identification was based on a multifractal spectrum calculated upon the fracture surfaces of an elastomeric material. This identification was carried out under various mechanical loading (stress level, frequency and loading ratio) and thermal damage.

The self-affinity nature of the surface roughness anisotropy is roughly identified through the power spectrum analysis. Then, the clustering singularity structure and its multiscaling characteristics are further examined with the box-counting technique and multifractal analysis.

According to the features extracted from the multifractal spectrum obtained, it is apparent that the fracture surface morphology exhibits fewer irregularities with the crack initiation, and more irregularities appear with the crack propagation, until the phase of the final rupture of the material which presents fewer irregularities. This identification is very useful to determine the fracture causes of manufactured parts from the studied material.
\end{abstract}

Keywords: surface roughness, multifractal, fracture, irregularities.

\section{Introduction}

The fracture surfaces contain the history of micro crack signatures, which are the indices of the fatigue of the studied materials. In the past few years, most commonly applied methods in a numerical description of fracture surfaces are quantitative fractography and image analysis $[1,2]$.

The concept of fractal geometry has been proved to be very useful in describing fracture morphology of materials. The Stach's works [3-6] have shown the effectiveness of multifractal analysis in the fractographic study. 
A lot of works have shown that a broad range of metal and ceramic materials exhibit a multifractal property on their fracture surface [7-9], but there are very few contributions to the fractographic study of elastomeric materials $[10,11]$. However, in the present work an investigation of the multifractal analysis for elastomeric material is presented.

This paper is organized as follows. In Section 2, we describe the mathematical foundation of multifractal analysis and the characteristics of the data used. In Section 3, we present the results obtained with the multifractal spectrum in the context of scanning electron microscope of fracture images, and then we discuss these results. We conclude our work in Section 4.

\section{Tools and research material}

\subsection{Data}

In this study, crack propagation tests using a Dynamic Mechanical Analyser (a TA instrument DMA 2980) have been carried out under various mechanical conditions (stress level, frequency and loading ratio) and thermal damage. For the crack propagation study, we used an edge cracked simple tension specimen, the elastomeric sample sizes are: $14 \mathrm{~mm}$ of length $\times 8 \mathrm{~mm}$ of width $\times 1.70 \mathrm{~mm}$ of thickness.

In order to make a global study of the surface roughness, we have digitized the fracture surface with a white light optical profiling using vertical scanning interferometer. An example of 3D and 1D fracture profiles is given by figure (1). The extraction process of the $1 \mathrm{D}$ profiles is carried out in the direction of the crack propagation.

The morphology developments of the fracture surface were examined by a JOEL JSM-6480 LV scanning electron microscope instrument. The acquisition is done with a secondary electron and high vacuum mode.

Some examples of fracture development images of the elastomeric studied material are presented in figure (2).

\subsection{Theory and methods}

Multifractal structure was first introduced by Mandelbrot [12], it is characterized by an infinite set of critical exponents describing the scaling of the moments of the distribution of some quantity. Since then, this feature has been observed in various objects, such as the energy dissipation set in turbulence [13], strang attractors in chaotic dynamical systems [14] and others. In our work, the multifractal analysis has been carried out using Legendre transform.

This method is based on the approach proposed by Stanczyk and Sharpe [15] for natural texture classification, in our application this method is applied to fracture texture identification. It uses a box-counting approach for determining fractal dimension, which estimates the probability $p_{i}(\varepsilon)$ of every single box that contain irregular mass (fracture patterns). 


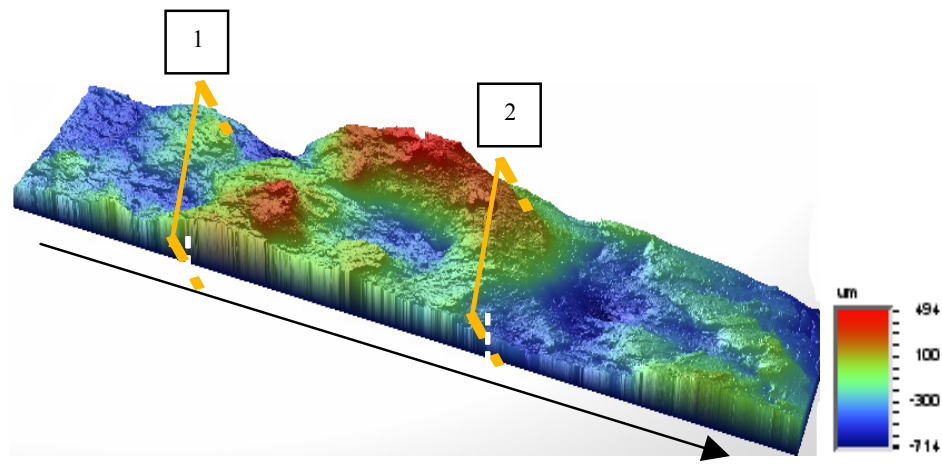

$-a-$
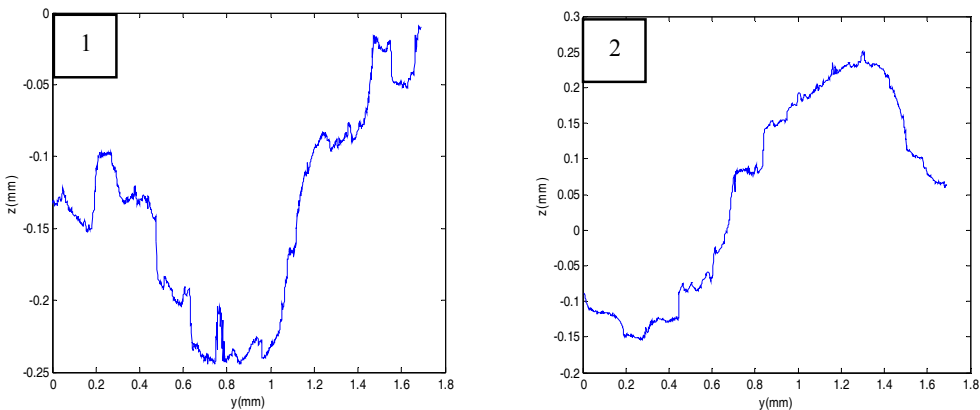

$-b-$

Figure 1: $\quad$ Example of digitized fracture surface. (a) 3D roughness profile, (b) two transverse sections.
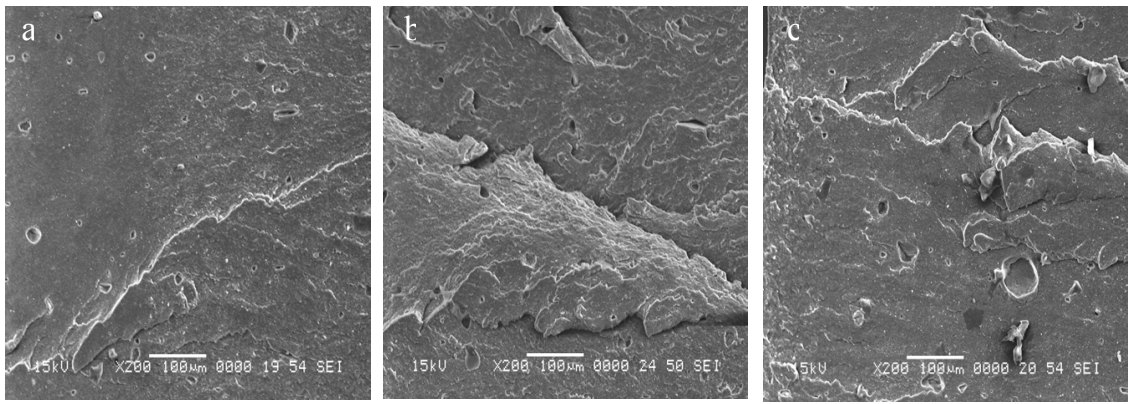

Figure 2: Fracture development SEM images: (a) crack initiation, (b) crack propagation and (c) final rupture. 
Doing so for SEM images of size $840 \times 840$ pixels, the images can be divided into many boxes of size $1 \times 1$, and let $\varepsilon=1 / \mathrm{L}(\varepsilon \rightarrow 0)(\mathrm{L}=840)$.

We briefly recall some basic facts about the multifractal theory. See also [16].

$\mu$ is defined as a Borel probability measure on $[0,1] \times[0,1]$. Let $v_{n}$ a linear size of "box" around $(i, j)$ upon which we evaluate $\mu$. We also define the subregion $I_{i, j, n}$ as follow:

$$
I_{i, j, n}=\left[\frac{i}{v_{n}}, \frac{i+1}{v_{n}}\right] \times\left[\frac{j}{v_{n}}, \frac{j+1}{v_{n}}\right]
$$

If we consider the quantity $\tau_{n}$ defined by the formula (2)

$$
\tau_{n}(q)=-\frac{\log \left(\sum_{i, j} \mu\left(I_{i, j, n}\right)^{q}\right)}{\log v_{n}}
$$

and if $\lim _{n \rightarrow \infty} \tau_{n}(q)=\tau(q)$, we can say that $\mu$ exhibits a multifractal behaviour.

With $q$ the moments order in a non-empty interval of $R$. So, $\tau(q)$ characterizes the global behaviour of the measure.

For the local structures, the Hölder exponent $\alpha$ at point $(i, j)$ is defined according to the formula (3)

$$
\alpha_{I_{i, j}}(q)=\frac{d \tau(q)}{d q}
$$

To obtain the Legendre multifractal spectrum $f(\alpha)$ associated with $\mu$, the Legendre transform is applied as follow:

$$
f(\alpha)=\inf _{q \in R}(q \alpha-\tau(q))
$$

During the data acquisition process, the images can be corrupted with noise, thus to remove this noise from the images we have used wavelets DWT-2D. Hence, the spectrum is calculated from the de-noised images.

\section{Analysis and results}

\subsection{Spectral analysis of fracture surface roughness anisotropy}

Spectral analysis provides an essential tool for understanding the frequency components of a surface roughness. It has been used to study different topics such as the upper and lower limits of fractal dimensions of a fracture surface and the selection of a proper sampling bandwidth for 3D surface topography measurement [17]. The method of power spectrum can simply be realized by means of the fast Fourier transformation and it leads to useful results [18].

As demonstrated in figure 3(a) the fracture surface under $20 \%-5 \mathrm{~Hz}$ displays a $f^{-8 / 3}$ power-law shape over a range of frequencies $(f=$ spatial frequency $\left.\left(\mathrm{mm}^{-1}\right)\right)$. This decrease of power-law corresponds to a scale-invariance by 
anisotropic transformation. This phenomenon is observed for all the fracture surfaces obtained from the four fracture conditions (figure 3(b)).

\subsection{Multifractal analysis}

The shape and the extension of the $f(\alpha)$-curve contains significant information about the distribution characteristics of the examined data set. In general, the spectrum has a concave downward curvature, with a range of $\alpha$ values increasing correspondingly to the increase in the heterogeneity of the distribution.

In our work, we analyse the fracture surface in order to identify the fracture morphology that we can find in the direction of the crack propagation. This identification is shown by three separate multifractal spectrums, the first one is estimated upon the crack initiation SEM images, the second one from the crack propagation SEM images, and the last one from the final rupture zone (see figure 4(a)).

This phenomena, is repeated for the four applied fracture conditions (see figures 4(a), 4(b), 4(c) and 4(d)). The width of each multifractal spectrum of all fracture conditions is reported in table 1 .

For the majority of the fracture conditions, we notice that the spectrum of the crack initiation zone is less wide than that calculated on the crack propagation zone, and wider than the spectrum of the final rupture zone. Therefore, we can say that, at the crack propagation zones the surface texture contains more irregularities.

The fracture surfaces of the studied material under different fracture conditions display distinct multifractal behaviours. This difference of behaviours was estimated by the correlation coefficient between the various obtained multifractal spectrums. The correlation coefficient $\mathrm{R}^{2}$ was calculated by comparing the various $f(\alpha)$ curves with a curve of the three zones (crack initiation, crack propagation and final rupture) obtained at the fracture condition of $20 \%-5 \mathrm{~Hz}$. The correlation results are shown in table 2 . We note that we note that the fracture surface at the condition of $20 \%-5 \mathrm{~Hz}-120^{\circ}$ presents a best correlation with a correlation coefficient equal to $0,9924(\sim 1)$. Therefore, the fracture patterns presented at the two zones are almost similar.

\section{Conclusion}

A new method of crack path identification has been developed by combining scanning electron microscopy, secondary electron signal and multifractal image analysis.

An advantage of the multifractal analysis methods is the possibility of taking into account the complicated fracture morphology development (crack initiation, crack propagation and final rupture). According to the multifractal spectrum results calculated upon the three zones at each fracture conditions, we conclude that the fracture structure at the crack propagation step displays more irregularities then other zones. 


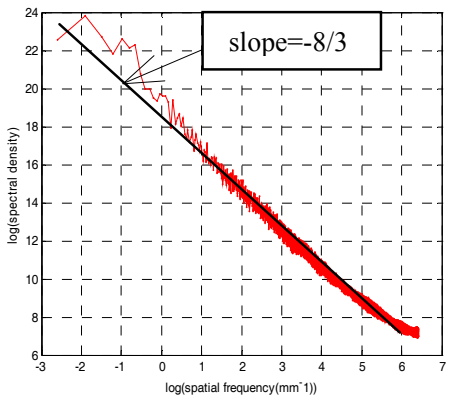

(a)

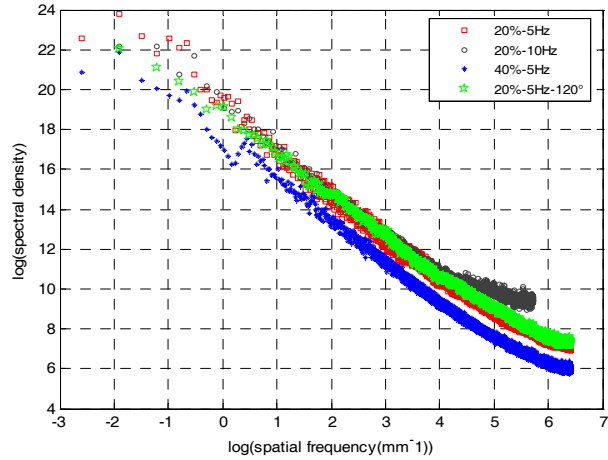

(b)

Figure 3: Log-log plot of the power spectral density of the fracture profiles resulting from: (a) $20 \%-5 \mathrm{~Hz}$ fracture condition only, (b) $20 \%-5 \mathrm{~Hz}$, $20 \%-10 \mathrm{~Hz}, 40 \%-5 \mathrm{~Hz}$ and $20 \%-5 \mathrm{~Hz}-120^{\circ}$ fracture conditions.

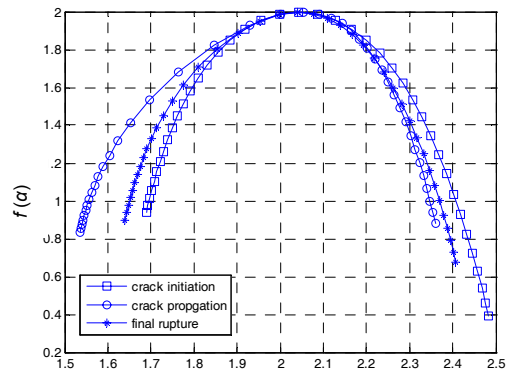

$(\mathrm{a})$

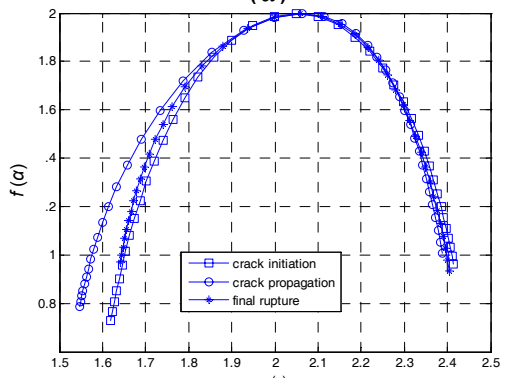

(c)

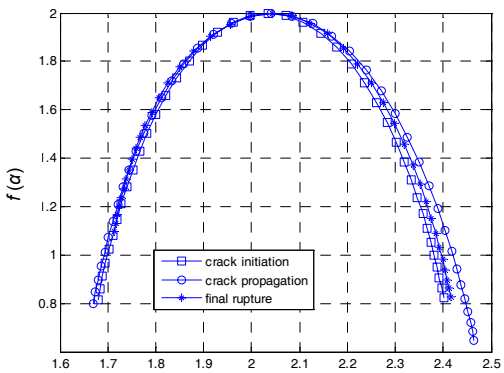

(b)

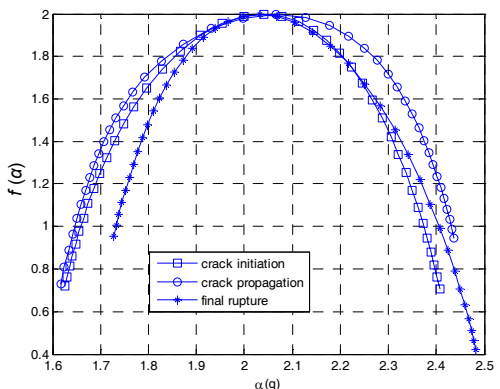

(d)

Figure 4: $\quad$ Multifractal spectrum of fracture surface for four different fracture conditions: a) $20 \%-5 \mathrm{~Hz}$, b) $20 \%-10 \mathrm{~Hz}$, c) $20 \%-5 \mathrm{~Hz}-120^{\circ}$, d) $40 \%$ $5 \mathrm{~Hz}$. 


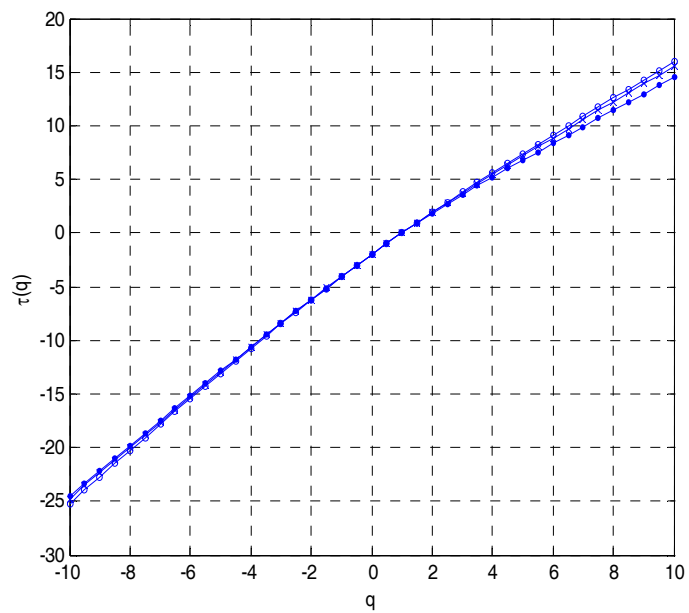

Figure 5: The relationships of slope $\tau_{q}$ with $q$.

Table 1: $\quad$ Fracture conditions and the width of the $f(\alpha)$ spectrum upon the crack path.

\begin{tabular}{|l|l|l|l|l|l|l|l|l|l|}
\hline \multirow{2}{*}{$\begin{array}{l}\text { Fracture } \\
\text { conditions }\end{array}$} & \multicolumn{3}{|l|}{ Crack initiation } & \multicolumn{3}{|c|}{ Crack propagation } & \multicolumn{3}{l|}{ Final rupture } \\
\cline { 2 - 10 } & $\alpha_{\max }$ & $\alpha_{\min }$ & $\Delta \alpha$ & $\alpha_{\max }$ & $\alpha_{\min }$ & $\Delta \alpha$ & $\alpha_{\max }$ & $\alpha_{\min }$ & $\Delta \alpha$ \\
\hline $20 \%-5 \mathrm{~Hz}$ & 2.4834 & 1.6891 & 0.7943 & 2.3600 & 1.5351 & 0.8249 & 2.4074 & 1.6404 & 0.7670 \\
\hline $20 \%-10 \mathrm{~Hz}$ & 2.4005 & 1.6789 & 0.7216 & 2.4643 & 1.6693 & 0.7950 & 2.4158 & 1.7127 & 0.7031 \\
\hline $\begin{array}{l}20 \%-5 \mathrm{~Hz}- \\
120^{\circ}\end{array}$ & 2.4137 & 1.6194 & 0.7943 & 2.3884 & 1.5479 & 0.8405 & 2.4055 & 1.6409 & 0.7646 \\
\hline $40 \%-5 \mathrm{~Hz}$ & 2.4073 & 1.6261 & 0.7812 & 2.4371 & 1.6168 & 0.8203 & 2.4833 & 1.7281 & 0.7552 \\
\hline
\end{tabular}

Table 2: Correlation coefficient between $f(\alpha)$ curves of two different fracture conditions.

\begin{tabular}{|l|c|c|c|}
\hline Fracture conditions & $\mathrm{R}_{\text {ci }}^{2}$ & $\mathrm{R}_{\mathrm{cp}}^{2}$ & $\mathrm{R}_{\text {fr }}^{2}$ \\
\hline $20 \%-5 \mathrm{~Hz}$ & 1 & 1 & 1 \\
\hline $20 \%-10 \mathrm{~Hz}$ & 0.9596 & 0.8647 & 0.9802 \\
\hline $20 \%-5 \mathrm{~Hz}-120^{\circ}$ & 0.8828 & 0.9924 & 0.9905 \\
\hline $40 \%-5 \mathrm{~Hz}$ & 0.9878 & 0.9491 & 0.9907 \\
\hline
\end{tabular}

$\mathrm{R}_{\text {ci }}^{2}$ : correlation coefficient between the crack initiation $f(\alpha)$ curves for two different fracture conditions.

$\mathrm{R}_{\mathrm{cp}}^{2}$ : correlation coefficient between the crack propagation $f(\alpha)$ curves for two different fracture conditions.

$\mathrm{R}_{\mathrm{fr}}^{2}$ : correlation coefficient between the final rupture $f(\alpha)$ curves for two different conditions.

Anisotropic and heterogeneous fractal properties of fracture surfaces of the elastomeric studied material are highlighted. 
The multifractal behaviour of fracture surface indicates that multifractal spectra carry much additional information on the fracture conditions and structural properties of fracture surfaces, which seems to be helpful in understanding structural phenomena of fracture surfaces.

\section{References}

[1] Wojnar, L., Quantitative fractography: basis and computer aid of investigations. Krakow: Cracow University of Technology; 1990.

[2] Wojnar, L., Image analysis: applications in materials engineering. CRC Press, 1999.

[3] Stach, S. \& Cybo, J., Multifractal description of fracture morphology: theoretical basis. Mater Charact'03; 51(1):79-86, 2003.

[4] Stach, S. and al, Multifractal description of fracture morphology: investigation of the fractures of sintered carbides. Mater Charact'03, 51(1):87-93, 2003.

[5] Stach, S. and al, Multifractal or fractographic fracture line description. Inz Mater'04; 140(3):440-3, 2004.

[6] Stach, S. \& Cybo, J., Multifractal detection of overlaps based on a stereometric analysis of fracture surface: assumptions. Materials Characterization'06; 56:449-53, 2006.

[7] Sim, B. L., Agterberg, P. and Beaudry, C., Determining the cutoff between background and relative base metal smelter contamination levels using multifractal methods. Computers \& Geosciences, Volume 25, Issue 9, pp. 1023-1041, 15 November 1999

[8] Jing, L.I. and al, Fractal analysis of crack paths in $\mathrm{Al}_{2} \mathrm{O}_{3}$-TiC-4\%Co composites. Transactions of Nonferrous Metals Society of China, Volume 16, Issue 4, pp. 795-799, August 2006.

[9] James, L. and al, Fracture surface examination of dental ceramics using fractal analysis. Dental Materials, Volume 21, Issue 6, pp. 586-589, June 2005.

[10] Jelcic, Z., Holjevac-Grguric, T. and Rek, V., Mechanical properties and fractal morphology of high-impact polystyrene/poly(styrene-b-butadieneb-styrene) blends. Polymer Degradation and Stability, Volume 90, Issue 2, pp. 295-302, November 2005.

[11] Ouahabi, A., AIT AOUIT, D., Fractales et ondelettes pour le signal et l'image (Chapter 3). Optimisation en traitement du signal et de l' image, ed. Hermes Lavoisier, Paris, pp. 73-104, 2006.

[12] Mandelbrot, B. B., Intermittent turbulence in self-similar cascades: Divergence of high moments and dimension of the carrier. J. Fluid Mech. 62 (1974), pp. 331-358, 1974.

[13] Frisch, U., and al, Turbulence and predictability in geophysical fluid dynamics and climate dynamics. International School of Physics Enrico Fermi, North Holland Amsterdam, 1985.

[14] Grassberger, P., Procacia, I., Measuring the strangeness of strange attractors. Physica. D, vol. 9, nº1-2, pp. 189-208, 1983. 
[15] Stanczyk, P. \& Sharpe, P., Classification of natural images from shape analysis of the Legendre multifractal spectrum. Fractals: Theory and applications in Engineering, Springer-Verlag, pp. 67-79 London, 1999.

[16] Jaffard, S., Construction de fonctions multifractales ayant un spectre de singularités prescrit. C.R. Acad. Sci. Paris, pages 19-24, T. 315, Series I, 1992.

[17] Lin, T.Y., Blunt, L. and Stout, K. J., Determination of proper frequency bandwidth for 3D topography measurement using spectral analysis. Wear. 166, 221-232, 1993.

[18] Russ, J.C., Fractal surfaces. Plenum Press, New York, 1994. 